\title{
EXTENSIONS OF VERMA MODULES
}

BY

KEVIN J. CARLIN

\begin{abstract}
A spectral sequence is introduced which computes extensions in category $\mathcal{O}$ in terms of derived functors associated to coherent translation functors.

This is applied to the problem of computing extensions of one Verma module by another when the highest weights are integral and regular. Some results are obtained which are consistent with the Gabber-Joseph conjecture. The main result is that the highest-degree nonzero extension is one-dimensional.

The spectral sequence is also applied to the Kazhdan-Lusztig conjecture and related to the work of Vogan in this area.
\end{abstract}

0. Introduction. In $\S 1$ the basic properties of category $\mathcal{O}$ are summarized. The notation is nearly standard, although the Bruhat order is the opposite to the usual definition on a Coxeter group.

$\S 2$ contains the general results on translation in category $\mathcal{O}$. The main results are 2.9 and 2.10. 2.9 computes the derived functors of a pair of adjoint functors. These are seen to compute extensions in $\mathcal{O}$ in terms of a spectral sequence, 2.10. In general this spectral sequence has at most two lines but (fortunately) collapses in important situations.

In $\$ 3$ these methods are applied to the problem of computing $\operatorname{Ext}_{o}^{*}\left(M_{x}, M_{y}\right)$ where $M_{x}$ is a Verma module with integral regular highest weight (parametrized by the Weyl group). One well-known result which is easily obtained is the SchmidDelorme-Casselman vanishing theorem (3.7). Another is part of the result of Gabber and Joseph on this problem (3.2(i)). Gabber and Joseph conjecture that the dimensions of the $\operatorname{Ext}_{o}^{*}\left(M_{x}, M_{y}\right)$ are given by the absolute values of the coefficients of the $R_{x, y}$ polynomials of [13], (3.1). The results obtained here are consistent with their conjecture and are aimed at proving it. The main result (3.8) is that

$$
\operatorname{dim} \operatorname{Ext}_{\mathcal{O}}^{l(x)-l(y)}\left(M_{x}, M_{y}\right)=1
$$

where $x \leqslant y$ in the Bruhat order. Two results are given which compute $\operatorname{Ext}^{*}\left(M_{x}, M_{y}\right)$ for special situations. For a general class of pairs, $(x, y)$, called Coxeter pairs the Gabber-Joseph conjecture is true (3.11). A lemma is given which computes $\operatorname{Ext}_{\mathscr{O}}^{1}\left(M_{x}, M_{y}\right)$ in certain cases (3.12). In particular this implies that the GabberJoseph conjecture is true when $l(x)-l(y) \leqslant 3(3.13)$.

In the last section the methods of $\$ 2$ are applied to the problem (which is part of the Kazhdan-Lusztig result) of computing $\operatorname{Ext}_{o}^{*}\left(M_{x}, L_{y}\right)$ where $L_{y}$ is the irreducible quotient of $M_{y}$. Again several well-known results are easily obtained $(4.2,4.3)$. Using

Received hy the editors October 19, 1983 and, in revised form, June 25, 1984.

1980 Mathematics Subject Classification. Primary 17B10: Secondary 17B55. 17B56. 
this method it is shown that

$$
\operatorname{dim} \operatorname{Ext}_{\mathscr{O}}^{l(x)-l(y)}\left(M_{x}, L_{y}\right)=1
$$

when $x \leqslant y$ (4.6) and the calculations in this case are seen to parallel those of Vogan.

1. Notations and preliminaries. Let $g$ be a finite-dimensional semisimple Lie algebra over $\mathbf{C}$. Let $b$ be a Borel subalgebra; $b=h \oplus \eta^{+}$, the Levi decomposition where $h$ is a Cartan subalgebra. Let $R, R^{+}, B$ (respectively) be the roots, positive roots, simple roots determined by $b$. In terms of the root spaces, $g^{\alpha}, \alpha \in R$ :

$$
\begin{gathered}
\eta^{+}=\sum_{\alpha \in R^{+}} \oplus g^{\alpha}, \quad \eta^{-}=\sum_{\alpha \in R^{+}} \oplus g^{-\alpha}, \\
g=\eta^{-} \oplus h \oplus \eta^{+} .
\end{gathered}
$$

Let $\rho=\frac{1}{2} \sum_{\alpha \in R^{\prime}} \cdot \alpha$. Let $\sigma$ be an involution of $g$ which fixes $h, \sigma\left(\eta^{+}\right)=\eta$. If $\alpha \in R$, let $\alpha^{2}=2 \alpha /(\alpha, \alpha)$ where $(-,-)$ denotes the Killing form.

Let $W$ denote the Weyl group of $g$. If $\alpha \in R$, let $s_{\alpha}$ denote the reflection

$$
s_{\alpha} \lambda=\lambda-\left(\lambda, \alpha^{\vee}\right) \alpha, \quad \lambda \in h^{*} .
$$

The pairing ( $\left.W, S=\left\{s_{\alpha}\right\}_{\alpha \in B}\right)$ is a Coxeter group.

Let $l(-)$ denote the length function. The Bruhat order on $W$ is the transitive closure of the relation, $x<y$, defined to hold if $l(x)=l(y)+1$ and $x^{-1} y=s_{\alpha}$, $\alpha \in R$. (This is the opposite of the usual Bruhat order.) Let $w^{0}$ denote the unique longest element and let $e$ be the identity. (For the properties of $<$ refer to [9].)

If $V$ is a $g$-module and $\lambda \in h^{*}$, let

$$
V^{\lambda}=\{v \in V: h \cdot v=\lambda(h) v, \text { all } h \in h\} .
$$

If $V^{\lambda} \neq 0, \lambda$ is called a weight of $V$. A vector which belongs to a maximal weight space (with respect to the ordering $\lambda \leqslant \mu$ if $\mu-\lambda \in \mathbf{Z}^{+} R^{+}$) is called a highest weight vector. Let $V^{(\hbar)}$ denote the sum of the finite-dimensional weight spaces of $V$. Let

$$
P=\left\{\lambda \in \hbar^{*}:\left(\lambda, \alpha^{\vee}\right) \in \mathbf{Z} \text { all } \alpha \in R^{+}\right\}
$$

be the integral weights. Define $P^{+}$and $P^{++}$, the dominant integral and regular dominant integral weights, by replacing $\mathbf{Z}$ by $\mathbf{Z}^{+}$and $\mathbf{N}$ respectively.

Definition 1.1. If $\lambda \in h^{*}$, the Verma module with highest weight, $\lambda-\rho$, is the induced module

$$
U(g) \underset{U(\ell)}{\otimes} \mathbf{C}_{\lambda-\rho}
$$

where $\mathbf{C}_{\lambda-\rho}$ is the one-dimensional $b$-module with the trivial $n^{+}$-action and weight, $\lambda-\rho$, and where $U(-)$ denotes the enveloping algebra.

$M(\lambda)$ is generated by a highest weight vector (unique up to scalar multiple) and is free as an $U\left(n^{-}\right)$-module. Also $M(\lambda)^{(\hbar)}=M(\lambda)$. $M(\lambda)$ has a unique irreducible quotient denoted $L(\lambda)$. In general this is infinite-dimensional but the finite-dimensional $g$-modules correspond to $P^{++}[10,7.2 .6]$. Let $E(\lambda)$ denote the finite-dimensional $g$-module whose highest weight is a conjugate of $\lambda$ under $W$. 
The natural setting for the study of Verma modules is category $\mathcal{O}$. For the definitions and properties of $\mathcal{O}$ the interested reader is referred to [3]. For convenience the more important information used here is summarized below.

The irreducibles of $\mathcal{O}$ are the $L(\lambda), \lambda \in h^{*}$. Each object has finite composition series, so $\mathcal{O}$ has the Krull-Schmidt property. Let $[V, L(\lambda)]$ denote the multiplicity if $V \in \mathcal{O}$.

Let $z(g)$ be the centre of $\mathscr{U}(g)$, and if $\lambda \in h^{*}$ let $\chi_{\lambda}$ denote the infinitesimal character associated to $\lambda[10,7.4 .7,7.4 .8]$. If $V \in \mathcal{O}$, let $P_{\lambda} V$ denote the generalized eigenspace of $z(\mathrm{~g})$ for the character $\chi_{\lambda}$. Then $P_{\lambda}$ is an exact functor on $\mathcal{O}$ and $P_{\lambda}=P_{\mu}$ if and only if $\lambda=w \cdot \mu, w \in W$.

If $Q$ is a set of representatives for $h^{*} / W$,

$$
V=\sum_{\lambda \in Q} \oplus P_{\lambda} V
$$

where only finitely many $P_{\lambda} V \neq 0$. In this way $\mathcal{O}=\amalg_{\lambda} \mathcal{O}_{\lambda}$ where $P_{\lambda}$ is the projection functor onto $\mathcal{O}_{\lambda}=P_{\lambda} \mathcal{O}$.

$\mathcal{O}$ is self-dual under the duality

$$
V^{\#}=\operatorname{hom}(V, \mathbf{C})^{(\hbar)}
$$

where $X \in g$ acts on $f \in \operatorname{hom}_{\mathbf{C}}(V, \mathbf{C})$ by $(X \cdot f)(v)=f(\sigma(X) \cdot v)$. This means $\left(V^{\#}\right)^{\#} \cong V$ and $\operatorname{hom}(V, W) \cong \operatorname{hom}\left(W^{\#}, V^{\#}\right)$ if $V, W \in \mathcal{O}$ (natural in $V$ and $W$ ). $L(\lambda)^{\#} \cong L(\lambda)$.

DEFINITION 1.2. $V \in \mathcal{O}$ admits $p$-filtration if there exists a sequence of submodules

$$
V=V_{1} \supseteq V_{2} \supseteq \cdots \supseteq V_{n+1}=(0)
$$

where $V_{i} / V_{i+1} \cong M\left(\lambda_{i}\right), i=1, \ldots, n$. Let $[V, M(\lambda)]$ be the multiplicity of $M(\lambda)$ in a $p$-filtration of $V .[V, M(\lambda)]$ is independent of the particular $p$-filtration [3, p. 89].

Proposition 1.3. $V$ admits $p$-filtration if and only if $V$ is a free $\mathscr{U}\left(n^{-}\right)$-module of finite rank with a basis of weight vectors.

COROLlaRY 1.4. Let $E$ be a finite-dimensional g-module. If $V \in \mathcal{O}$ admits p-filtration then so does $V \otimes E$. In that case, $[V \otimes E, M(\mu)]=\sum \operatorname{dim} E^{\lambda}[V, M(\mu-\lambda)]$.

$\mathcal{O}$ has enough projectives and injectives. It is enough to give the indecomposables. Let $P(\lambda)$ denote the unique indecomposable projective with irreducible quotient, $L(\lambda)$. Each $P(\lambda)$ admits $p$-filtration and $[P(\lambda), M(\mu)]=[M(\mu), L(\lambda)]$ (the "duality" theorem, [3, Proposition 2, part 2]). The indecomposable injectives are the $I(\lambda)=P(\lambda)^{\#}$. Ext* will always refer to Ext* ${ }_{\mathscr{o}}^{*}$.

2. Translation functors and duality. The application of translation functors to representation theory was initiated by Zuckermann and Jantzen $[\mathbf{1 2 , 2 0 ]}$. The aim of this section is to introduce certain derived functors which will compute extensions in 0 .

To begin recall the basic properties of translation functors. 
Definition 2.1. Let $\lambda-\mu \in P$. Define

$$
T_{\lambda}^{\mu}=P_{\mu} \circ(-\otimes E(\mu-\lambda)) \circ P_{\lambda}
$$

where $E(\mu-\lambda)$ is the finite-dimensional $g$-module with extreme weight, $\mu-\lambda$.

Proposition 2.2. Let $\lambda-\mu \in P$.

(i) $T_{\lambda}^{\mu}$ is an exact functor with left (right) adjoint $T_{\mu}^{\lambda}$.

(ii) There is a natural isomorphism from $T_{\lambda}^{\mu} \circ \#$ to \#० $T_{\lambda}^{\mu}$.

(iii) If $V \in \mathcal{O}$ admits p-filtration then so does $T_{\lambda}^{\mu} V$.

Proof. (i) $T_{\lambda}^{\mu}$ is exact because it is a composition of exact functors. If $V$ and $W \in \mathcal{O}$, there is a sequence of natural isomorphisms, $\operatorname{hom}\left(T_{\lambda}^{\mu} V, W\right) \rightarrow \operatorname{hom}\left(P_{\lambda} V \otimes E, P_{\mu} W\right) \rightarrow \operatorname{hom}\left(P_{\lambda} V, E^{*} \otimes P_{\mu} W\right) \rightarrow \operatorname{hom}\left(V, T_{\mu}^{\lambda} W\right)$.

(ii) Clearly projection commutes with duality. The result follows from the natural isomorphism

$$
\operatorname{hom}(V \otimes E, \mathbf{C})^{(h)} \cong \operatorname{hom}(V, \mathbf{C})^{(\hbar)} \otimes E .
$$

(iii) Because $T_{\lambda}^{\mu}$ is exact the result follows from 1.4 and [3, Lemma 1].

In the case that $\lambda$ and $\mu$ lie in the same closed chamber, the results of Jantzen allow one to compute the Verma-module multiplicities of $T_{\lambda}^{\mu} V$ under the conditions of 2.2(iii). Because the Verma modules of $\mathcal{O}_{\lambda}$ are the $\{M(w \lambda)\}_{{ }^{*} \in W}$, it is enough to compute $\left[T_{\lambda}^{\mu} M(w \lambda): M\left(w^{\prime} \mu\right)\right], w, w^{\prime} \in W$.

Proposition 2.3 (Jantzen, [12, 2.9]). Let $\lambda$ and $\mu \in P$ lie in the same closed chamber. Let $w$ and $w^{\prime} \in W$.

(i) $\left[T_{\lambda}^{\mu} M(w \lambda), M\left(w^{\prime} \mu\right)\right] \neq 0 \Rightarrow w^{\prime} \mu=w \sigma \mu$, where $\sigma \in W^{\lambda}$ (the stabilizer of $\lambda$ in $W)$.

(ii) If $\sigma \in W^{\lambda},\left[T_{\lambda}^{\mu} M(w \lambda), M(w \sigma \lambda)\right]=1$.

In the remainder of this section fix a pair $(\lambda, \mu)$ with $\lambda-\mu \in P$. In the proofs the pair $(\lambda, \mu)$ may be suppressed.

Let $S_{\lambda}^{\mu}=T_{\mu}^{\lambda} \circ T_{\lambda}^{\mu}$ be the "coherent" translation functor. From 2.2 it is easily seen that $S_{\lambda}^{\mu}$ is an exact, selfadjoint functor which commutes with \#.

It will be convenient to give a duality operation on functors and natural transformations. If $F$ is a functor and $\mathcal{N}$ is a natural transformation (from $G$ to $H$ ) then ${ }^{\#} F=\# \circ F \circ \#$ and ${ }^{\#} \mathscr{N}=\# \circ \mathscr{N} \circ \#$ is a natural transformation from ${ }^{\#} H$ to ${ }^{\#} G$. For example, ${ }^{\#} S_{\lambda}^{\mu} \cong S_{\lambda}^{\mu}$.

Definition 2.4. If $V \in \mathcal{O}$, let $I_{\lambda}^{\mu} V$ be the map from $V$ to $S_{\lambda}^{\mu} V$ which is the image of the identity under the isomorphism

$$
\operatorname{hom}\left(T_{\lambda}^{\mu} V, T_{\lambda}^{\mu} V\right) \stackrel{\sim}{\rightarrow} \operatorname{hom}\left(V, S_{\lambda}^{\mu} V\right) \text {. }
$$

The general properties of these maps were developed by Gabber and Joseph in [11]. In particular $I_{\lambda}^{\mu}$ can be seen to be a natural transformation from Id to $S_{\lambda}^{\mu}[11$, 5.1.4]. Note that under natural identifications the dual transformation ${ }^{*} I_{\lambda}^{\mu}(V)$ is the image of the identity under the isomorphism

$$
\operatorname{hom}\left(T_{\lambda}^{\mu} V, T_{\lambda}^{\mu} V\right) \stackrel{\sim}{\rightarrow} \operatorname{hom}\left(S_{\lambda}^{\mu} V, V\right) \text {. }
$$


These maps will be used to define a pair of dual functors in the following way. Definition 2.5. Let $C_{\lambda}^{\mu}=$ Coker $I_{\lambda}^{\mu}$, that is, for $V \in \mathcal{O}, C_{\lambda}^{\mu} V$ satisfies the criterion

$$
V \rightarrow S_{\lambda}^{\mu} V \rightarrow C_{\lambda}^{\mu} V \rightarrow 0 \text { is exact. }
$$

Coker $I_{\lambda}^{\mu}$ is a natural transformation from $S_{\lambda}^{\mu}$ to $C_{\lambda}^{\mu}$. Note that ${ }^{\#} C_{\lambda}^{\mu} \cong \operatorname{Ker}{ }^{\#} I_{\lambda}^{\mu}$.

Proposition 2.6. $\left(C_{\lambda}^{\mu},{ }^{\#} C_{\lambda}^{\mu}\right)$ is an adjoint pair of functors with $C_{\lambda}^{\mu}$ right exact and ${ }^{\#} C_{\lambda}^{\mu}$ left exact.

Proof. Let $0 \rightarrow V^{\prime \prime} \rightarrow V \rightarrow V^{\prime} \rightarrow 0$ be exact. Then

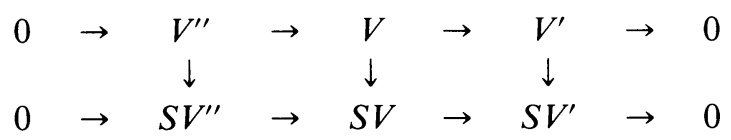

commutes and has exact rows. The "snake" lemma implies that $C_{\lambda}^{\mu}$ is right exact. Clearly ${ }^{\#} C_{\lambda}^{\mu}$ is left exact.

If $V, W \in \mathcal{O}$, consider the diagram:

$$
\begin{aligned}
& 0 \rightarrow \operatorname{hom}(C V, W) \rightarrow \underset{\uparrow}{\operatorname{hom}(S V, W)} \rightarrow \underset{\uparrow}{\operatorname{hom}(V, W)} \\
& 0 \rightarrow \operatorname{hom}\left(V,{ }^{\#} C W\right) \rightarrow \operatorname{hom}(V, S W) \rightarrow \operatorname{hom}(V, W)
\end{aligned}
$$

The rows are exact since hom(-, - ) is left exact and the square commutes because of $[11,5.1 .4]$. By diagram chasing and the naturality of the diagram, restriction gives a natural isomorphism.

The natural problem at this point is to compute the derived functors. In order to do this the crucial step is to find sufficient conditions on $V \in \mathcal{O}$ so that $I_{\lambda}^{\mu} V$ is an injective map, that is, so that

$$
0 \rightarrow V \rightarrow S_{\lambda}^{\mu} V \rightarrow C_{\lambda}^{\mu} V \rightarrow 0
$$

is an exact sequence.

Proposition 2.7. If $V \in \mathcal{O}_{\lambda}$ is free as a $\mathscr{U}\left(n^{-}\right)$-module, then $I_{\lambda}^{\mu} V$ is injective.

Proof. Let $K=\operatorname{Ker} I_{\lambda}^{\mu} V$. Then from the exact sequence $0 \rightarrow K \stackrel{k}{\rightarrow} V \stackrel{I V}{\rightarrow} S V$ one obtains the commutative diagram:

$$
\begin{array}{ccc}
\operatorname{hom}(T V, T V) & \stackrel{\operatorname{hom}}{\rightarrow} V, S V) \\
\downarrow & & \downarrow \\
\operatorname{hom}(T K, T V) & \stackrel{\operatorname{hom}}{\rightarrow}(K, S V) .
\end{array}
$$

Taking the image of the identity in hom $(T V, T V)$, one has $T(k)$ is the zero map. Because $T$ is exact, this means $T_{\lambda}^{\mu} K=0$. Suppose that $K$ is nonzero. Any vector belonging to a maximal weight space of $K$ generates a Verma module, say $M(w \lambda)$, $w \in W$, because $V$ is free as a $\mathscr{U}\left(n^{-}\right)$-module. However it is clear that $T_{\lambda}^{\mu} M(w \lambda) \neq 0$. In fact, 1.4 implies that $M(w \mu)$ occurs in the $p$-filtration of $T_{\lambda}^{\mu} M(w \lambda)$. This contradicts the fact that $T_{\lambda}^{\mu} K=0$ since $T_{\lambda}^{\mu}$ is exact.

COROLlaRY 2.8. (i) If $V \in \mathcal{O}_{\lambda}$ admits p-filtration then $I_{\lambda}^{\mu} V$ is an injective map. 
(ii) If $P \in \mathcal{O}_{\lambda}$ is projective then

$$
0 \rightarrow P \rightarrow S_{\lambda}^{\mu} P \rightarrow C_{\lambda}^{\mu} P \rightarrow 0 \text { is exact } .
$$

(iii) If $I \in \mathcal{O}_{\lambda}$ is injective then

$$
0 \rightarrow{ }^{\#} C_{\lambda}^{\mu} I \rightarrow S_{\lambda}^{\mu} I \rightarrow I \rightarrow 0 \text { is exact }
$$

Proof. (i) is obvious, because of 1.3. (ii) follows because every projective admits p-filtration [3, Proposition 2]. Because the dual of a projective is injective, (iii) is the dual of (ii).

These properties permit one to calculate the derived functors of $C_{\lambda}^{\mu}$ and ${ }^{\sharp} C_{\lambda}^{\mu}$.

THEOREM 2.9.

$$
\begin{aligned}
L^{p} C_{\lambda}^{\mu}= & \left(\begin{array}{ll}
\text { Coker } I_{\lambda}^{\mu} & \text { if } p=0, \\
\operatorname{Ker} I_{\lambda}^{\mu} & \text { if } p=1, \\
0 & \text { otherwise, }
\end{array}\right. \\
R^{p}\left[{ }^{\#} C_{\lambda}^{\mu}\right] & =\left(\begin{array}{ll}
\operatorname{Ker}^{\#} I_{\lambda}^{\mu} & \text { if } p=0, \\
\text { Coker }^{\#} I_{\lambda}^{\mu} & \text { if } p=1, \\
0 & \text { otherwise. }
\end{array}\right.
\end{aligned}
$$

ProOF. Let $P^{*}$ be a projective resolution of $V \in \mathcal{O}_{\lambda}$. Then, because of 2.8(ii),

$$
0 \rightarrow P^{*} \rightarrow S P^{*} \rightarrow C P^{*} \rightarrow 0
$$

is an exact sequence of complexes. By elementary homological algebra, the associated long exact sequence collapses because $S$ is exact and yields

$$
\begin{gathered}
0 \rightarrow L^{1} C_{\lambda}^{\mu} V \rightarrow V \rightarrow S_{\lambda}^{\mu} V \rightarrow C_{\lambda}^{\mu} V \rightarrow 0 \text { and } \\
0 \rightarrow L^{p} C_{\lambda}^{\mu} V \rightarrow 0, \quad \text { if } p>1 .
\end{gathered}
$$

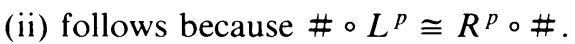

Note that this recovers the ignored functors, $\operatorname{Ker} I_{\lambda}^{\mu}$ and Coker ${ }^{\#} I_{\lambda}^{\mu}$.

Finally we can give the main result of this section.

THEOREM 2.10. Let $V, W$ be modules in $\mathcal{O}_{\lambda}$.

(i) If ${ }^{\#} I_{\lambda}^{\mu} W$ is onto, then there is a spectral sequence of term

$$
E_{2}^{p . q}=\operatorname{Ext}^{p}\left(L^{q-p} C_{\lambda}^{\mu} V, W\right) \Rightarrow \operatorname{Ext}^{q}\left(V,{ }^{*} C_{\lambda}^{\mu} W\right) .
$$

(ii) If $I_{\lambda}^{\mu} V$ is an injective map, then there is a spectral sequence of term

$$
E_{2}^{p, q}=\operatorname{Ext}^{p}\left(V, R^{q-p}\left[{ }^{\#} C_{\lambda}^{\mu}\right] W\right) \Rightarrow \operatorname{Ext}^{q}\left(C C_{\lambda}^{\mu} V, W\right) .
$$

REMARKS. Because of 2.9 these spectral sequences are at worst two-line spectral sequences. Also the condition in 2.10(ii) is satisfied by any Verma module.

Proof. If $F=C_{\lambda}^{\mu}, G=\operatorname{hom}(-, W)$ then (i) is the Grothendieck spectral sequence for $G \circ F$. By $2.6, R^{q}(G \circ F) V \cong \operatorname{Ext}^{q}\left(V,{ }^{\#} C_{\lambda}^{\mu} W\right)$. To prove (i) it suffices to show that if $P$ is projective then

$$
\operatorname{Ext}^{i}\left(C_{\lambda}^{\mu} P, W\right)=0 \quad \text { for } i>0 .
$$

Start by noting that

$$
0 \rightarrow P \stackrel{I P}{\rightarrow} S P \stackrel{\varepsilon}{\rightarrow} C P \rightarrow 0
$$


is a projective resolution of $C_{\lambda}^{\mu} V$. Hence the complex

$$
0 \rightarrow \operatorname{hom}(S P, W) \rightarrow \operatorname{hom}(P, W) \rightarrow 0
$$

computes the extension groups of interest. But we have a commutative diagram [11, 5.4.1]:

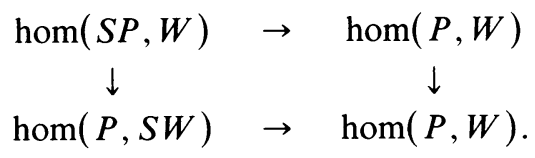

Because $P$ is projective and ${ }^{\#} I_{\lambda}^{\mu} W$ is onto, the bottom map is onto. Therefore the top map is onto and $\operatorname{Ext}^{1}(C P, W)=0$. It is clear that the higher extension groups are zero.

(ii) is ${ }^{\#}(\mathrm{i})$.

3. The Gabber-Joseph conjecture. Fix a weight, $\lambda \in P^{++}$. Let $M_{x}$ denote $M(x \lambda)$, if $x \in W$ (similarly define $L_{x}$ and $P_{x}$ ). The translation principle states that the nonnegative integers

$$
\operatorname{dim} \operatorname{Ext}^{q}\left(M_{x}, M_{y}\right) ; \quad x, y \in W,
$$

are independent of the particular choice of $\lambda \in P^{++}$. This is easily seen from 2.2(i) and 2.3 .

In [11], Gabber and Joseph suggest the following conjecture for these integers:

Conjecture 3.1 [11, 5.2.4]. If $x, y \in W$ then

$$
\operatorname{dim} \operatorname{Ext}^{q}\left(M_{x}, M_{y}\right)=r_{q}(x, y),
$$

where the $r_{q}(x, y)$ are uniquely defined by the following recursion:

$$
r_{0}(x, y)=\zeta(x, y)=\left(\begin{array}{ll}
1 & \text { if } x \leqslant y \\
0 & \text { otherwise }
\end{array}\right.
$$

Let $x s>x, s \in S$.

$$
r_{q}(x, y)=\left(\begin{array}{ll}
r_{q}(x s, y s) & \text { if } y s>y, \\
r_{q}(x s, y)+r_{q-1}(x s, y)-r_{q-1}(x s, y s) & \text { if } y s<y .
\end{array}\right.
$$

Remark. These are the absolute values of the coefficients of the polynomials, $R_{x, y} ; x, y \in W$, introduced in [13]. Their main result in this direction is

Proposition 3.2 [11, 5.2.1 and 5.2.3]. Let $x s>x$ and $y s<y$ where $x, y \in W$ and $s \in S$.

(i) $\operatorname{Ext}^{q}\left(M_{x s}, M_{y}\right) \cong \operatorname{Ext}^{q}\left(M_{x}, M_{y s}\right)$.

(ii)

$$
\begin{aligned}
& \operatorname{dim} \operatorname{Ext}^{q}\left(M_{x}, M_{y}\right) \geqslant \operatorname{dim} \operatorname{Ext}^{q}\left(M_{x s}, M_{y}\right)+\operatorname{dim} \operatorname{Ext}^{q-1}\left(M_{x s}, M_{y}\right) \\
& -\operatorname{dim} \operatorname{Ext}^{q-1}\left(M_{x s}, M_{y s}\right) \text {. }
\end{aligned}
$$

The Gabber-Joseph conjecture would be proved if there were equality in (ii). 
In this section the spectral sequence of 2.10 is applied to this problem. First we specialise 2.10 and give the following notation:

Definition 3.3. Let $s=s_{\alpha} \in S, \alpha \in B$. Choose $\mu_{\alpha} \in P$ so that $\left(\lambda-\mu_{\alpha}, \beta\right) \geqslant 0$ for $\beta \in B$ and $\left(\lambda-\mu_{\alpha}, \beta\right)=0$ implies that $\beta=\alpha$. Define

$$
\begin{aligned}
& \theta_{s}=S_{\lambda}^{\lambda-\mu_{\alpha}}, \quad I^{s}=I_{\lambda}^{\lambda-\mu_{\alpha}}, \\
& { }^{s} \theta={ }^{\#} C_{\lambda}^{\lambda-\mu}, \quad{ }^{s} I={ }^{\#} I_{\lambda}^{\lambda-\mu}, \\
& \theta^{s}=C_{\lambda}^{\lambda-\mu_{\alpha}} .
\end{aligned}
$$

( $\theta_{s}$ is referred to as coherent translation "through" the $\alpha$-root wall in the sense that $\theta_{s}$ is thought of as taking $\mathcal{O}_{\lambda}$ to $\mathcal{O}_{s \lambda}$.)

The form of 2.10 which will be used in the remainder of this article is the following specification of 2.10(ii).

Proposition 3.4. Let $x \in W, s \in S$. If $N \in \mathcal{O}_{\lambda}$ then there is a spectral sequence

$$
E_{2}^{p, q}=\operatorname{Ext}^{p}\left(M_{x}, R^{q-p}\left[{ }^{s} \theta\right] N\right)
$$

converging to $\operatorname{Ext}^{q}\left(\theta^{s} M_{x}, N\right)$.

Proof. From 2.8, $I^{s} M_{x}$ is injective. So the condition of 2.10 (ii) is satisfied.

To apply this result one must know the derived functors of $\boldsymbol{\theta}^{s}$ and ${ }^{s} \boldsymbol{\theta}$ on Verma modules in $\mathcal{O}_{\lambda}$. These are all computable.

Proposition 3.5. Let $x \in W, s \in S$ and $x s>x$.

$$
\begin{aligned}
R^{p}\left[{ }^{s} \theta\right] M_{x} & =\left(\begin{array}{ll}
M_{x s} & \text { if } p=0, \\
0 & \text { otherwise, }
\end{array}\right. \\
L^{p} \theta^{s} M_{x s} & =\left(\begin{array}{ll}
M_{x} & \text { if } p=0, \\
0 & \text { otherwise. }
\end{array}\right.
\end{aligned}
$$

Proof. By $[11,3.13]$ there is an exact sequence

$$
0 \rightarrow M_{x s} \stackrel{I^{s} M_{v s}}{\rightarrow} \theta_{s} M_{x} \stackrel{s}{\rightarrow} M_{x} \rightarrow 0
$$

The result follows from 2.9 .

In order to apply 3.4 only a little more is needed.

Proposition 3.6. Let $x \in W, s \in S$ and $x s<x$.

$$
R^{p}\left[{ }^{s} \theta\right] M_{x}=\left(\begin{array}{ll}
M_{x} & \text { if } p=0, \\
M_{x} / M_{x s} & \text { if } p=1, \\
0 & \text { otherwise. }
\end{array}\right.
$$

Proof. Because $\theta_{s} M_{x} \cong \theta_{s} M_{x s}[\mathbf{1 1}, 3.6]$, there is a commutative diagram

$$
\begin{aligned}
& 0 \rightarrow M_{x s} \rightarrow M_{x} \rightarrow M_{x} / M_{x s} \rightarrow 0 \\
& 0 \rightarrow \theta_{s} M_{x s} \rightarrow \theta_{s} M_{x} \rightarrow \rightarrow 00 \quad \rightarrow 0
\end{aligned}
$$

with exact rows. By the snake lemma and 2.9, ${ }^{s} \theta M_{x s} \cong{ }^{s} \theta M_{x}=M_{x}$ and $R^{1}\left[{ }^{s} \theta\right] M_{x} \cong$ $M_{v} / M_{x}$. 
Let $x, y \in W, s \in S$ with $x s>x$. Now the spectral sequence of 3.4 becomes

$$
E_{2}^{p, q}=\operatorname{Ext}^{p}\left(M_{x s}, R^{q-p}\left[{ }^{s} \theta\right] M_{y}\right) \Rightarrow \operatorname{Ext}^{q}\left(M_{x}, M_{y}\right)
$$

because of 3.5(ii). This provides an "inductive step" for calculating $\operatorname{Ext}^{q}\left(M_{x}, M_{y}\right)$.

Under these conditions, consider the case $y s>y$. 3.5(i) implies that $E_{2}^{q . q}=$ $\operatorname{Ext}^{4}\left(M_{x s}, M_{y s}\right)$ and all other terms are zero. Because the bidegree of $d_{r}$ is $(r, 1)$, $E_{2}=E_{\infty}$ and 3.2(i) is an immediate consequence.

If $y s<y$ the situation is more complicated. From 3.6 one obtains

$$
E_{2}^{q-1, q}=\operatorname{Ext}^{q-1}\left(M_{x s} / M_{y s}\right), \quad E_{2}^{q, q}=\operatorname{Ext}^{q}\left(M_{x s}, M_{y}\right)
$$

and all other terms are zero. Schematically,

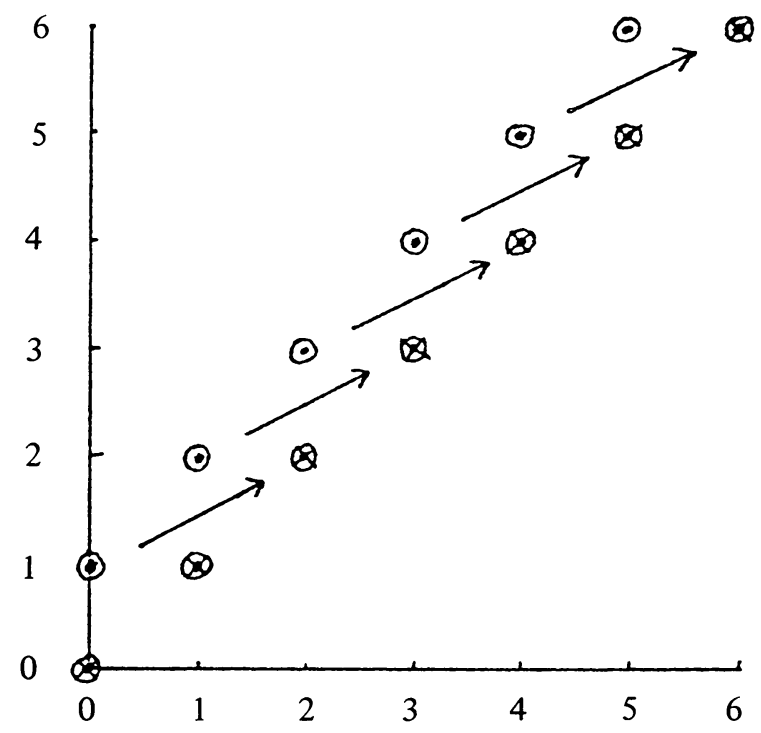

represents $E_{2}^{p, q}$, where the arrows represent the only nontrivial coboundary maps

$$
d_{2}^{q-1 . q}: \operatorname{Ext}^{q-1}\left(M_{x s}, M_{y} / M_{y s}\right) \rightarrow \operatorname{Ext}^{q+1}\left(M_{x s}, M_{y}\right) .
$$

In order to compute one must determine this map. It may be conjectured that $d_{2}$ factors as

$$
\operatorname{Ext}^{q-1}\left(M_{x s}, M_{y} / M_{y s}\right) \stackrel{\delta_{1}}{\rightarrow} \operatorname{Ext}^{q}\left(M_{x s}, M_{y s}\right) \stackrel{\delta_{2}}{\rightarrow} \operatorname{Ext}^{q+1}\left(M_{x s}, M_{y}\right),
$$

where $\delta_{1}$ and $\delta_{2}$ are the coboundaries of the obvious long-exact sequences.

Lacking the above determination one can still prove many of the well-known results on extensions in this setting. 3.2(i) has already been obtained above as a trivial consequence. As another application one can prove the vanishing theorem of Delorme and Schmid.

Proposition $3.7\left[15,5.4-6 ; 8\right.$, Theorem 4]. Let $x$ and $y \in W$. If $\operatorname{Ext}^{q}\left(M_{x}, M_{y}\right) \neq 0$ then $x \leqslant y$ and $0 \leqslant q \leqslant l(x)-l(y)$. 
Proof (Induction on $l(x)$ ). By the duality theorem $M_{e}=P_{e}$. If $\operatorname{Ext}^{q}\left(M_{e}, M_{y}\right) \neq 0$ then $q=0$ and clearly $y=e$. If $l(x)>0$, choose $s \in S$ so that $x s>x$. Suppose that $\operatorname{Ext}^{q}\left(M_{x}, M_{y}\right) \neq 0$. There are two cases to consider.

If $y s>y$, then $\operatorname{Ext}^{q}\left(M_{x s}, M_{y s}\right) \cong \operatorname{Ext}^{q}\left(M_{x}, M_{y}\right)$. The induction hypothesis implies that $x s \leqslant y s$ and $0 \leqslant q \leqslant l(x)-l(y)$, but $x s \leqslant y s$ if and only if $x \leqslant y$.

If $y s<y$, consider the spectral sequence $(*)$. Clearly either $E_{2}^{q-1, q} \neq 0$ or $E_{2}^{q, q} \neq 0$. This means that one of $\operatorname{Ext}^{q}\left(M_{x s}, M_{y}\right), \operatorname{Ext}^{q}\left(M_{x s}, M_{y s}\right)$ or $\operatorname{Ext}^{q-1}\left(M_{x s}, M_{y}\right)$ is nonzero. In each case the induction hypothesis implies that $x \leqslant y$ and $0 \leqslant q \leqslant l(x)$ $-l(y)$.

The next result is the main result of this section. It says that the last nonzero Ext ${ }^{4}$ is one-dimensional.

TheOREM 3.8. Let $x, y \in W$. If $x \leqslant y$ then

$$
\operatorname{dim} \operatorname{Ext}^{l(x)-l(y)}\left(M_{x}, M_{y}\right)=1 .
$$

Proof (Induction on $l(x)$ ). As in 3.7 the case $x=e$ is trivial. Choose $s \in S$ so that $x s>x$.

If $y s>y, \operatorname{Ext}^{q}\left(M_{x}, M_{y}\right) \cong \operatorname{Ext}^{q}\left(M_{x s}, M_{y s}\right)$ and the result follows from the induction hypothesis.

If $y s<y$ consider $(*)$. Let $n=l(x)-l(y)$. Because of $3.7, E_{2}^{p . q}=0$ if $q>n$ and one has

$$
\begin{gathered}
E_{2}^{n-1, n}=\operatorname{Ext}^{n-1}\left(M_{x s}, M_{y} / M_{y s}\right) \cong \operatorname{Ext}^{n-1}\left(M_{x s}, M_{y}\right), \\
E_{2}^{n, n}=\operatorname{Ext}^{n}\left(M_{x s}, M_{y}\right)=0 .
\end{gathered}
$$

Since the bidegree of $d_{2}$ is $(2,1)$, $\operatorname{Ext}^{n}\left(M_{x}, M_{y}\right) \cong \operatorname{Ext}^{n-1}\left(M_{x s}, M_{y}\right)$.

REMARK. The referee points out that this result and 4.6 are easily seen to be equivalent and that the latter result can be proved using the BGG resolution and duality. Also the above proof avoids using 3.2(ii) (which implies that dim $\left.\operatorname{Ext}^{l(x)-l(y)}\left(M_{x}, M_{y}\right) \geqslant 1\right)$ because it is not necessary to this result and one hopes that 3.2(ii) can be recovered in this context.

It can easily be seen from [8, Theorem 2] using the Euler characteristic that

$$
\sum_{i}(-1)^{i} \operatorname{dim} \operatorname{Ext}^{i}\left(M_{x}, M_{y}\right)=\delta_{x, y} .
$$

In particular when $l(x)-l(y)=2, x<y, 3.8$ implies that

$$
\begin{aligned}
& \operatorname{dim} \operatorname{Ext}^{0}\left(M_{x}, M_{y}\right)=1, \\
& \operatorname{dim} \operatorname{Ext}^{1}\left(M_{x}, M_{y}\right)=2, \\
& \operatorname{dim} \operatorname{Ext}^{2}\left(M_{x}, M_{y}\right)=1 .
\end{aligned}
$$

One can extend this result in two ways. In the remainder of this section these are considered.

Proposition 3.9. Let $x s>x, y s<y$ where $x, y \in W$ and $s \in S$. If $x s \nless y s$ then $\operatorname{dim} \operatorname{Ext}^{q}\left(M_{x}, M_{y}\right)=\operatorname{dim} \operatorname{Ext}^{q}\left(M_{x s}, M_{y}\right)+\operatorname{dim}^{\operatorname{Ext}^{q-1}}\left(M_{x s}, M_{y}\right)$. 
Note. This also follows from [11, 5.2.3].

Proof. Because $x s \nless y s$, one has

$$
\operatorname{Ext}^{q}\left(M_{x s}, M_{y} / M_{y s}\right) \cong \operatorname{Ext}^{q}\left(M_{x s}, M_{y}\right)
$$

It follows from $(*)$ that

$$
\operatorname{dim} \operatorname{Ext}^{q}\left(M_{x}, M_{y}\right) \leqslant \operatorname{dim} \operatorname{Ext}^{q}\left(M_{x s}, M_{y}\right)+\operatorname{dim} \operatorname{Ext}^{q-1}\left(M_{x s}, M_{y}\right) .
$$

The opposite inequality is 3.2 (ii).

Together with the recursion of 3.1 this results allows the computation of $\operatorname{Ext}^{*}\left(M_{x}, M_{v}\right)$ for certain pairs $(x, y)$.

Definition 3.10. A pair $(x, y) ; x, y \in W$ is called a Coxeter pair if $r_{1}(x, y)=$ $l(x)-l(y)$.

Example. Suppose that $x=s_{1} \cdots s_{n}$ where the $s_{i}$ are distinct in $S, l(x)=n$. Then for any $y \geqslant x,(x, y)$ is a Coxeter pair.

The recursion of 3.1 in the case $q=1, x s>x, x$ and $y \in W, s \in S$ is

$$
r_{1}(x, y)=\left(\begin{array}{ll}
r_{1}(x s, y s) & \text { if } y s>y, \\
r_{1}(x s, y) & \text { if } y s<y, x s \leqslant y s, \\
r_{1}(x s, y)+1 & \text { if } y s<y, x s \nless y s .
\end{array}\right.
$$

First note that $r_{1}(x, y) \leqslant l(x)-l(y)$ so that $(x, y)$ is Coxeter when $r_{1}(x, y)$ is maximal. In the situation $x s>x, y s<y,(x, y)$ is Coxeter if and only if $(x s, y)$ is Coxeter and $x s \nless y s$. The following result is therefore a trivial consequence of 3.9.

Proposition 3.11. Let $x$ and $y \in W$. If $(x, y)$ is a Coxeter pair then

$$
\operatorname{dim} \operatorname{Ext}^{q}\left(M_{x}, M_{y}\right)=r_{q}(x, y)=\left(\begin{array}{l}
n \\
q
\end{array}\right),
$$

where $n=l(x)-l(y)$.

The following lemma again gives a special situation in which equality holds in 5.2(ii).

Lemma 3.12. Let $x s>x, y s<y$ where $x, y \in W$ and $s \in S$. If $\left[M_{y}, L_{x s}\right]=$ $\left[M_{y s}, L_{x s}\right]$ then

$$
\operatorname{Ext}^{1}\left(M_{x}, M_{y}\right) \cong \operatorname{Ext}^{1}\left(M_{x s}, M_{y}\right)
$$

Proof. The sequence

$$
0 \rightarrow \operatorname{hom}\left(P_{x s}, M_{y s}\right) \rightarrow \operatorname{hom}\left(P_{x s}, M_{y}\right) \rightarrow \operatorname{hom}\left(P_{x s}, M_{y} / M_{y s}\right) \rightarrow 0
$$

is exact. Because $\operatorname{dim} \operatorname{hom}\left(P_{x s}, V\right)=\left[V, L_{x s}\right]$ the hypothesis implies that $\operatorname{hom}\left(P_{x s}, M_{y} / M_{y s}\right)=0$ hence $\operatorname{hom}\left(M_{x s}, M_{y} / M_{y s}\right)=0$. The result follows from (*).

One can use this result to compute $\operatorname{Ext}^{1}\left(M_{x}, M_{y}\right)$ for low rank (where the multiplicities are known). More generally because Jantzen has shown that $\left[M_{y}, L_{x}\right]$ $\leqslant 1$ if $l(x)-l(y) \leqslant 2, x, y \in W[12,5.2 .3]$ the following result is a trivial consequence of 3.12 . 
Proposition 3.13. Let $x, y \in W$. If $l(x)-l(y) \leqslant 3$ then

$$
\operatorname{dim} \operatorname{Ext}^{q}\left(M_{x}, M_{y}\right)=r_{q}(x, y)
$$

Proof (Induction on $l(x)$ ). Assume $x \leqslant y$. The case $x=e$ is trivial. Let $x s>x$, $s \in S$. There are three cases to be considered:

I. $y s>y$.

II. $y s<y, x s \nless y s$.

III. $y s<y, x s \leqslant y s$.

Cases I and II are handled easily by 3.2(i), 3.9 and the induction hypothesis. In the remaining case, $l(x)-l(y)=3$ and one must show that $\operatorname{dim} \operatorname{Ext}^{q}\left(M_{x}, M_{y}\right)=$ $\operatorname{dim} \operatorname{Ext}^{4}\left(M_{x s}, M_{y}\right)$. Because of 3.8 and the remarks preceding 3.9 it suffices to show this for $q=1$. But in this case $\left[M_{v}, L_{x s}\right]=\left[M_{v s}, L_{x s}\right]=1$ and the result follows by 3.12 and the induction hypothesis.

It may be hoped that $(*)$ can provide a proof of equality in 3.2(ii). This would seem to depend on the determination of $d_{2}$ and would perhaps not go beyond [11, 3.2.3] where a necessary and sufficient condition is given.

4. The irreducible case. In this section the problem of computing

$$
\operatorname{Ext}^{*}\left(M_{x}, L_{y}\right), \quad x, y \in W,
$$

is considered. This problem is solved in terms of the Kazhdan-Lusztig conjecture [13, Conjecture 1.5] which has recently been proved by Brylinski-Kashiwara [5] and independently by Bernstein-Beilinson [1]. Of the equivalent forms of the KazhdanLusztig conjecture given by Vogan [18], the most interesting (from this viewpoint) is the following "parity" vanishing conjecture.

Conjecture 4.1 (Kazhdan - LuSzTig - Vogan).

$$
\operatorname{Ext}^{q}\left(M_{x}, L_{y}\right) \neq 0 \Rightarrow q \equiv l(x)-l(y) \bmod 2 .
$$

The truth of this conjecture entails the truth of all the other equivalent statements given in [13]. In particular, the dimensions of the above extensions are given by the coefficients of the $P_{x, y}$ polynomials.

The methods of $\$ 2$ can be applied to this problem. Let $x \in W, s \in S, x s>x$. Then the spectral sequence of 2.10(ii) (with the notation of $\S 3$ ) yields

$$
E_{2}^{p, q}=\operatorname{Ext}^{q}\left(M_{x s}, R^{q-p}\left[{ }^{s} \theta\right] L_{y}\right) \Rightarrow \operatorname{Ext}^{q}\left(M_{x}, L_{y}\right) .
$$

Obviously to use this to calculate one must compute $R^{p}\left[{ }^{s} \theta\right] L_{y}, y \in W$.

If $y s<y$ for $s \in S$, then $\theta_{s} L_{y}=0$ since $L_{y}$ is a quotient of $M_{y} / M_{y s}$ and $\theta_{s}$ is exact. This means ${ }^{s} I L_{y}$ is the zero map. By 2.9 this implies that

$$
R^{p}\left[{ }^{s} \theta\right] L_{y}=\left(\begin{array}{ll}
0 & \text { if } p=0 \\
L_{y} & \text { if } p=1, \\
0 & \text { otherwise }
\end{array}\right.
$$

Assuming that $x s>x, x \in W$, the spectral sequence $(\dagger)$ gives

$$
E_{2}^{q-1, q}=\operatorname{Ext}^{q-1}\left(M_{x s}, L_{y}\right)
$$

and all other terms are zero. Since this converges to $\operatorname{Ext}^{q}\left(M_{x}, L_{y}\right)$ the following result is immediate. 
Proposition 4.2. Let $s \in S$. Suppose that $x s>x$ and $y s<y$ where $x, y \in W$. Then

$$
\operatorname{Ext}^{q-1}\left(M_{x s}, L_{y}\right) \cong \operatorname{Ext}^{q}\left(M_{x}, L_{y}\right) .
$$

This result can be obtained by various other methods and has the easy corollary:

Corollary 4.3 (BotT's THeOREM).

$$
\operatorname{dim} \operatorname{Ext}^{q}\left(M_{x}, L_{e}\right)=\delta_{q, l(x)} .
$$

PRoof. If $x=e, M_{e}=P_{e}$ is projective and $\operatorname{hom}\left(M_{e}, L_{e}\right)$ is one-dimensional. If $x<e$, suppose $x s>x$ where $s \in S$. By the proposition, $\operatorname{Ext}^{q-1}\left(M_{x s}, L_{e}\right) \cong$ $\operatorname{Ext}^{4}\left(M_{x}, L_{e}\right)$. The result follows by induction on $l(x)$.

In order to calculate further one needs some description of $R^{p}[s \theta] L_{y}$ where $y s>y$. Because $\theta_{s} L_{y} \neq 0[11,3.6]$ the map ${ }^{s} I L_{y}$ is onto. Theorem 2.9 implies that $R^{p}[s \theta] L_{y}=0$ if $p>0$. It remains to determine ${ }^{s} \theta L_{y}$.

It is well known (cf. [17]) that the sequence

$$
0 \rightarrow L_{y} \stackrel{I^{s} L_{y}}{\rightarrow} \theta_{s} L_{y} \stackrel{s}{\rightarrow} L_{y} L_{y} \rightarrow 0
$$

is a complex if $y s>y$. Therefore ${ }^{s} \theta L_{y} / L_{y}$ can be identified with $\mathscr{U}_{\alpha} L_{y}$ (notation [18]) where $s=s_{\alpha}$ simply because this is defined as the cohomology of this complex. Both Vogan and Gabber-Joseph have studied the irreducible multiplicities of $\mathscr{U}_{\alpha} L_{y}$. Clearly this gives corresponding information for ${ }^{s} \theta L_{v}$.

Proposition 4.4 [11, 3.11]. Let $y s>y$ where $y \in W$ and $s \in S$.

(i) $\left[s \theta L_{y}, L_{v}\right]=1$,

(ii) $\left[s \theta L_{y}, L_{y s}\right]=1$,

(iii) If $\left[s \theta L_{v}, L_{\sigma}\right] \neq 0$ and $\sigma \neq y$, ys then
(a) $\sigma s<\sigma$,
(b) $\sigma<y s$,
(c) $l(\sigma)>l(y)$.

The only assertions of 4.4 which are not direct translations from [11, 3.11] are (i) and (iii)(b). They are also easily seen to be consequent with the aid of the following lemma which may be of independent interest.

LemMA 4.5. Let $y s>y$ where $y \in W, s \in S$. If $\sigma \in W$ then

$$
\left[{ }^{s} \theta L_{y}, L_{\sigma}\right] \leqslant\left[M_{y}, L_{\sigma}\right]+\left[M_{y s}, L_{\sigma}\right]-\delta_{\sigma, y} .
$$

In particular $\left[{ }^{s} \theta L_{y}, L_{y}\right] \leqslant 1,\left[{ }^{s} \theta L_{y}, L_{y s}\right] \leqslant 1$ and $\sigma \leqslant y s$ which provides the remainder of 4.4 in light of [11, 3.11].

Proof. Recall that $\left[V, L_{\sigma}\right]=\operatorname{dim} \operatorname{hom}\left(P_{\sigma}, V\right)$ if $V \in \mathcal{O}$ [3, Proposition 1]. Because $\left(\theta^{s},{ }^{s} \theta\right)$ is an adjoint pair of functors,

$$
\left[{ }^{s} \theta L_{y}, L_{\sigma}\right]=\operatorname{dim} \operatorname{hom}\left(P_{\sigma},{ }^{s} \theta L_{y}\right)=\operatorname{dim} \operatorname{hom}\left(\theta^{s} P_{\sigma}, L_{y}\right) .
$$

As in the proof of 2.10 the sequence

$$
0 \rightarrow \operatorname{hom}\left(\theta^{s} P_{\sigma}, L_{y}\right) \rightarrow \operatorname{hom}\left(\theta_{s} P_{\sigma}, L_{y}\right) \rightarrow \operatorname{hom}\left(P_{\sigma}, L_{y}\right) \rightarrow 0
$$


is exact. Hence it suffices to show that

$$
\operatorname{dim} \operatorname{hom}\left(\theta_{s} P_{\sigma}, L_{y}\right) \leqslant\left[M_{y}, L_{\sigma}\right]+\left[M_{y s}, L_{\sigma}\right] .
$$

Using 2.3 and the duality theorem it is easy to see that $\theta_{s} P_{\sigma}$ has $p$-filtration by $\left\{M_{\tau}\right\}_{\tau \in W}$ with multiplicities

$$
\left[\theta_{s} P_{\sigma}, M_{\tau}\right]=\left[P_{\sigma}, M_{\tau}\right]+\left[P_{\sigma}, M_{\tau s}\right]=\left[M_{\tau}, L_{\sigma}\right]+\left[M_{\tau s}, L_{\sigma}\right] .
$$

Proceeding as in [8, Lemma 2], $\boldsymbol{\theta}_{s} P_{\boldsymbol{\sigma}}$ is a quotient of

$$
\sum_{\tau \in W} \oplus P_{\tau}^{\left[M_{\tau}, L_{\sigma}\right]+\left[M_{\tau s} \cdot L_{\sigma}\right]}
$$

Since $\operatorname{dim}\left(P_{\tau}, L_{v}\right)=\delta_{\tau, y}$ this proves the lemma.

Using 4.4 and the spectral sequence $(\dagger)$ one can compute $\operatorname{Ext}^{*}\left(M_{x}, L_{y}\right), x$, $y \in W$, in certain cases. Below it is proved that

$$
\operatorname{dim} \operatorname{Ext}^{l(x)-l(y)}\left(M_{x}, L_{y}\right)=1 \text { if } x \leqslant y
$$

in this context. As pointed out in the remark following 3.8 , this is equivalent to 3.8 .

THEOREM 4.6. If $x, y \in W$ and $x \leqslant y$ then

$$
\operatorname{dim} \operatorname{Ext}^{l(x)-l(y)}\left(M_{x}, L_{y}\right)=1 .
$$

Proof. If $x=e$, the result is trivial because $M_{e}$ is projective. If $x<e$, let $s \in S$ such that $x s>x$. Let $n=l(x)-l(y)$. If $y s<y$, Proposition 4.2 implies that $\operatorname{Ext}^{n-1}\left(M_{x s}, L_{y}\right) \cong \operatorname{Ext}^{n}\left(M_{x}, L_{y}\right)$. If $y s>y$,

$$
0 \rightarrow L_{y} \rightarrow{ }^{s} \theta L_{y} \rightarrow W \rightarrow 0
$$

is exact.

From the corresponding long exact sequence (and the vanishing results)

$$
\operatorname{Ext}^{n}\left(M_{x s},{ }^{s} \theta L_{y}\right) \cong \operatorname{Ext}^{n}\left(M_{x s}, W\right) .
$$

In the same way it is easy to see from 4.4 that

$$
\operatorname{Ext}^{n}\left(M_{x s}, W\right) \cong \operatorname{Ext}^{n}\left(M_{x s}, L_{y s}\right) .
$$

The spectral sequence (7) in this case yields

$$
\operatorname{Ext}^{n}\left(M_{x}, L_{y}\right) \cong \operatorname{Ext}^{n}\left(M_{x s},{ }^{s} \theta L_{y}\right) \cong \operatorname{Ext}^{n}\left(M_{x s}, L_{y s}\right) .
$$

Thus in either case the result follows by induction on $l(x)$.

One can obtain a result analogous to 3.9 in the same way but this requires a slightly restrictive hypothesis on the structure of ${ }^{s} \theta L_{y}$ where $y s>y$ and will not be given.

The above results suggest that with more information about ${ }^{s} \theta L_{y}$ there is some hope of proving the parity-vanishing theorem 4.1. At least the method of 2.10 supplies an appropriate framework in which both the Gabber-Joseph and KazhdanLusztig conjectures could be considered. 


\section{REFERENCES}

1. A. A. Beilinson and I. N. Bernstein, Localisation of g-modules, C. R. Acad. Sci., Paris Sér. 292 (1981). 15-18.

2. I. N. Bernstein, I. M. Gelfand and S. I. Gelfand, Differential operators on the base affine space and a study of g-modules, Lie Groups and their Representations (Ed., I. M. Gelfand), Wiley, New York, 1975. pp. 39-64.

3. A category of g-modules, Funct. Anal. Appl. 10 (1976), 87-92.

4. N. Bourbaki, Groupes et algèbres de Lie, Chapters 4-6, Hermann, Paris, 1968.

5. J. Brylinski and M. Kashiwara, Kazhdan-Lusztig conjecture and holonomic systems, Invent. Math. 64 (1981). 387-410.

6. H. Cartan and S. Eilenberg, Homological algebra, Princeton Univ. Press, Princeton, N. J., 1956.

7. C. Curtis and J. Reiner, Representation theory of finite groups and associative algebras, Interscience, New York, 1962.

8. P. Delorme, Extensions dans la categorie $\mathcal{O}$ de Bernstein-Gelfand-Gelfand: Applications, preprint, Palaiscau, 1978.

9. V. V. Deodhar, Some characterizations of Bruhat ordering on a Coxeter group and determination of the relative Moehius function, Invent. Math. 39 (1977), 187-198.

10. J. Dixmier, Algèbres enveloppantes, Gauthier-Villars, Paris, 1974.

11. O. Gabber and A. Joseph, Towards the Kazhdan-Lusztig conjecture, Ann. Sci. Ecole Norm. Sup. 14 (1981), 261-302.

12. J. C. Jantzen, Moduln mit einem höchsten Gewicht, Lecture Notes in Math., vol. 750, Springer-Verlag, Berlin. 1980

13. D. Kazhdan and G. Lusztig, Representations of Coxeter groups and Hecke algebras, Invent. Math. $\mathbf{5 3}$ (1979), 165-184.

14. Schubert varieties and Poincare duality, Proc. Sympos. Pure Math., vol. 36, Amer. Math. Soc., Providence, R. I., 1980, pp. 185-203.

15. W. Schmid, Vanishing theorems for Lie algebra cohomology and the cohomology of discrete subgroups of semisimple Lie groups, Adv. in Math. 41 (1981), 78-113.

16. D. N. Verma, Structure of certain induced representations of complex semisimple Lie algebras, Bull. Amer. Math. Soc. 74 (1968), 160-166.

17. Moebius inversion for the Bruhat ordering on a Weyl group, Ann. Sci. Ecole Norm. Sup. 4 (1971), 393-398.

18. D. Vogan, Irreducible characters of semisimple Lie groups. I, Duke Math. J. 46 (1979), 61-108. Irreducible characters of semisimple Lie groups. II: the Kazhdan-Lusztig conjectures, Duke Math. J. 46 (1979), 805-859.

20. G. Zuckerman, Tensor products of finite and infinite dimensional representations of semisimple Lie groups. Ann. of Math. 106 (1977), 295-308.

Department of Mathematics, University of Essex, Wivenhoe Park, Colchester CO4 3SQ, ENGI.AND

Current address: Mathematics Institute, University of Warwick, Coventry CV4 7AL, England 